\title{
Malaria por Plasmodium vivax y falla al tratamiento radical
}

\author{
Julián García, Agustín Seijo, Andrés Benchetrit, Esteban Couto, \\ Sofía Echazarreta, Susana Lloveras y Tomás Orduna
}

\footnotetext{
Hospital de Enfermedades Infecciosas "Dr. Francisco Javier Muñiz", Buenos Aires, Argentina. Servicio de Medicina Tropical Patología Regional (CEMPRA-MT)

Los autores declaran no tener conflicto de interés Sin fuentes de financiamiento.

Recibido: 12 de enero de 2016 Aceptado: 8 de junio de 2016

Correspondencia a: Julián L. García julianlgarcia87@gmail.com
}

\section{Failure to radical cure in Plasmodium vivax malaria}

Relapsing Plasmodium vivax malaria is due to activation of dormant intrahepatic parasitic forms known as hypnozoits. Primaquine is the only available drug effective against hypnozoits and, alongside a schizonticidal drug, constitutes the radical treatment of malaria. Failure of radical treatment is frequently attributed to inadequate dosing, poor adherence, or reinfection. However, several cases of radical treatment failure without these factors have been reported, inferring that metabolic properties of the host or tolerance mechanisms of the parasite may be implied. A case of malaria due to Plasmodium vivax acquired in the Amazonic region, treated outside endemic area, with multiple relapses despite adequate radical treatment is described.

Key words: Plasmodium vivax, primaquine, relapse, treatment failure.

Palabras clave: Plasmodium vivax, primaquina, recaída, falla del tratamiento.

\section{Introducción}

$P$ lasmodium vivax ( $P$. vivax) es el agente etiológico de la malaria de mayor distribución geográfica, con 2,85 billones de personas en riesgo en todo el mundo ${ }^{1}$. La infección por $P$. vivax lleva a la formación de hipnozoítos que permanecen quiescentes en los hepatocitos del hospedero, pudiendo reactivarse meses o años luego de resuelto el cuadro clínico ${ }^{2}$.

Primaquina (PQ) es el único fármaco en uso para el tratamiento de la fase hepática de la infección y, en asociación con un agente esquizonticida, constituye el tratamiento radical del paludismo por $P$. vivax. Primaquina también está indicada como parte del tratamiento de la malaria por Plasmodium falciparum debido a su acción gametocida $^{3}$. Si bien su mecanismo de acción no se conoce completamente, los productos del metabolismo hepático del fármaco producirían un daño oxidativo del parásito ${ }^{4}$.

La falla al tratamiento radical, definida como la reaparición de la parasitemia luego del uso de PQ más un agente esquizonticida, puede explicarse por la administración de dosis subóptimas, una adherencia inadecuada al medicamento, o una reinfección ${ }^{5}$. Sin embargo, se han descrito casos de falla en que estos factores se encuentran ausentes $^{6-9}$. Se han postulado otras causas que pueden ser propias del hospedero o del parásito.

Asimismo, la efectividad de PQ varía en distintas regiones geográficas. En las regiones tropicales del Sudeste Asiático se han reportado mayores tasas de falla terapéutica, siendo hasta diez veces mayor en Tailandia, en comparación con India y Brasil ${ }^{10}$. En Sudamérica también se han comunicado casos de falla al tratamiento radical ${ }^{11}$. Duarte y cols. notificaron una tasa de falla de
$14 \%$ en pacientes con tratamiento supervisado en una zona no endémica de Brasil ${ }^{12}$. En la región amazónica de Brasil se ha descrito $5-10 \%$ de recaídas, posiblemente secundarias a una falla terapéutica en temporada de baja incidencia de la infección ${ }^{13}$.

La falla al tratamiento radical de la infección por $P$. vivax y las dificultades para identificar su causa constituyen un escenario complejo. Comunicamos un caso clínico de malaria por $P$. vivax con recaídas posteriores pese al tratamiento radical adecuado.

\section{Caso clínico}

Paciente de sexo masculino de 25 años residente en la provincia de Buenos Aires, con diagnóstico de paludismo después de tres meses de un viaje a la región amazónica de Bolivia, Perú, Ecuador y Brasil, tratado con mefloquina en otra institución. El paciente no regresó a área endémica.

Tres meses después de completado el tratamiento consultó en nuestro Servicio por presentar fiebre y cefalea de dos días de evolución. Al examen físico se constató un peso corporal de $72 \mathrm{~kg}$, palidez generalizada y esplenomegalia leve. Entre los análisis de laboratorio destacaba una leucocitosis de $14.300 / \mathrm{mm}^{3}$ con una fórmula leucocitaria conservada, plaquetas $84.000 / \mathrm{mm}^{3}$. La función renal y hepática se encontraba normal. Se realizó un frotis de sangre periférica (FSP) observándose trofozoítos compatibles con $P$. vivax, con una parasitemia $<2 \%$. El diagnóstico fue realizado en el Laboratorio de Referencia en Parasitología del Hospital de Enfermedades Infecciosas “Francisco J. Muñiz". Recibió tratamiento con cloroquina (CQ) $1.500 \mathrm{mg}$ de dosis total, constatándose 
una remisión clínica y parasitemia negativa a las $48 \mathrm{~h}$. El nivel de glucosa-6-fosfato deshidrogenasa fue de 266 $\mathrm{mg} / \mathrm{dl}$ (rango normal), por lo que recibió PQ $15 \mathrm{mg}$ al día durante 15 días, con buena adherencia. Se realizaron parasitemias de control en la primera y cuarta semana después del tratamiento resultando negativas.

Posteriormente presentó dos nuevos episodios de recaída por $P$. vivax. Siete meses después consultó por fiebre de cuatro días de evolución asociada a calofríos, cefalea holocraneana, artralgias y dolor abdominal. Al examen físico se observó palidez de piel y mucosas, ictericia en conjuntivas y petequias en miembros inferiores. En los exámenes de laboratorio se objetivó un hemograma y recuento plaquetario normales, y una bilirrubina de $2,5 \mathrm{mg} / \mathrm{dl}$ de predominio indirecto. El FSP informó una parasitemia $<2 \%$. Completó tratamiento con CQ 1.500 $\mathrm{mg}$ de dosis total y PQ $30 \mathrm{mg}$ al día durante 15 días, con una buena adherencia y parasitemia negativa a las cuatro semanas.

Cinco meses después consultó por presentar fiebre intermitente de dos días de evolución asociada a cefalea y astenia. El examen físico y el laboratorio no presentaron hallazgos patológicos. Se constató una parasitemia $<2 \%$. Reinició el mismo esquema con buena adherencia y con parasitemia negativa a las cuatro semanas. No presentó nuevas recaídas durante los 15 meses de seguimiento.

\section{Discusión}

La falla terapéutica, definida como una recaída luego del tratamiento radical, puede deberse a una reinfección, adherencia inadecuada a los medicamentos o por una dosificación subóptima ${ }^{5}$, las cuales fueron descartadas en nuestro paciente. Se han descrito casos similares, pudiendo atribuirse la recaída a parámetros farmacocinéticos de PQ o a un fenómeno de tolerancia del parásito.

Primaquina es una 8-aminoquinolina surgida hace más de 60 años. Es el único fármaco en uso para la erradicación de los hipnozoítos y, por consiguiente, para la prevención de recaídas en infecciones por $P$. vivax ${ }^{14-17}$. Su metabolismo involucra diferentes enzimas hepáticas del citocromo P-450 (CYP) como CYP 3A4, 2D6, 2B6, y del grupo de las mono-amino oxidasas (MAO) ${ }^{18}$. Si bien el perfil metabólico de PQ no se ha dilucidado en su totalidad, sus metabolitos provocarían una lisis parasitaria por medio de radicales libres del oxígeno y disrupción en la cadena respiratoria ${ }^{4}$. Asimismo, estos productos metabólicos se asocian a los efectos adversos hematológicos como metahemoglobinemia ${ }^{19} \mathrm{y}$ anemia hemolítica en pacientes con déficit de la enzima glucosa-6P-deshidrogenasa ${ }^{20}$. Por otra parte, el metabolismo del hospedero podría interferir con la eficacia de la PQ. Bennet y cols. tipificaron el fenotipo del CYP 2D6 en 25 pacientes, asociando cinéticas de metabolismo intermedio y lento con falla al tratamiento radical $^{21}$.

La Organización Mundial de la Salud (OMS) recomienda un régimen de PQ $15 \mathrm{mg}$ al día $(0,25 \mathrm{mg} / \mathrm{kg})$ durante 14 días para el tratamiento de las formas hepáticas de $P$. vivax, considerando ampliar a $30 \mathrm{mg}$ al día $(0,5 \mathrm{mg} /$ $\mathrm{kg}$ ) en pacientes con malaria adquirida en zonas tropicales, particularmente en el Sudeste Asiático, Indonesia u Oceanía ${ }^{3}$. Sin embargo, las guías americanas y británicas recomiendan una dosis de $30 \mathrm{mg}$ al día durante 14 días independiente del lugar de adquisición ${ }^{22-23}$. En un estudio retrospectivo, Townell y cols. evaluaron 143 episodios de recaída de malaria por $P$. vivax provenientes de distintas zonas geográficas, en una región no endémica, asociando un régimen de $30 \mathrm{mg}$ al día por 14 días (dosis total $\geq 420 \mathrm{mg}$ ) a una tasa de recaídas de $1,9 \%$ versus $18 \%$ en pacientes con dosificaciones menores ${ }^{7}$. En contraposición, Rajgor y cols. evaluaron en forma prospectiva la tasa de recaída de 1.252 pacientes infectados en India, aleatorizados en un grupo control y tres esquemas de tratamiento, 15 $\mathrm{mg}$ al día por 14 días, $30 \mathrm{mg}$ al día por siete días y $30 \mathrm{mg}$ al día por 14 días, sin encontrar diferencias significativas entre dichos regímenes ${ }^{24}$.

En 1944 se describió la cepa Chesson, resistente a pamaquina (8-aminoquinolina) y tolerante a regímenes de $15 \mathrm{mg}$ al día de PQ. Algunos autores atribuyen su surgimiento al uso masivo de atebrina (derivado sintético de la quinina) y pamaquina, dos fármacos relacionados estructuralmente con PQ, por las fuerzas armadas que participaron en la Segunda Guerra Mundial en el Pacífico Sur. Desde entonces, se han reportado distintos casos de falla al tratamiento radical. Desafortunadamente, hasta el momento no existen marcadores diagnósticos de resistencia a PQ en hipnozoítos ${ }^{4}$.

Bright y cols. realizaron la genotipificación de cepas de $P$. vivax de tres aislados en un paciente con malaria adquirida en Sudán con múltiples recaídas, pese al tratamiento radical, fuera de zona endémica ${ }^{25}$, demostrando la presencia de mutaciones puntuales en regiones homólogas a los genes de $P$. falciparum asociados a resistencia a fármacos antimaláricos: $p v d h p s, p v m d r 1, p v m r p, p v d h f r$ y $p v d h p s^{26-28}$. Entre ellos, el gen $p v m r p$ es de particular interés, ya que codifica una proteína probablemente asociada a la resistencia a múltiples fármacos antipalúdicos, relacionada funcionalmente con el eflujo de glutatión. Este gen podría intervenir en un mecanismo de resistencia a $\mathrm{PQ}^{29}$. Estudios in vitro han demostrado que parásitos knock out para pvmrp incrementan significativamente su sensibilidad a diferentes fármacos antimaláricos, incluyendo $\mathrm{PQ}^{30}$. Además, se encontró una similitud entre el perfil genómico de estos aislados con la cepa Brazil-I, asociada clínicamente a tolerancia a $\mathrm{PQ}^{31}$, y diferencias significativas con la cepa Sal-I, sensible a PQ ${ }^{25}$. En consecuencia, la mayor tasa de falla al tratamiento radical 
en determinadas áreas geográficas podría explicarse por la presencia de cepas de $P$. vivax tolerantes a la $\mathrm{PQ}^{32}$. En nuestro paciente no fue posible realizar estudios fenotípicos ni genotípicos en la cepa de $P$. vivax aislada.

\section{Conclusiones}

La OMS recomienda el uso de PQ 0,25 $\mathrm{mg} / \mathrm{kg}$ al día por 14 días para cepas de áreas templadas y $0,5 \mathrm{mg} /$ $\mathrm{kg}$ al día por igual intervalo de tiempo para cepas de áreas tropicales, como del este de Asia y Oceanía. Esta recomendación se basa en estudios experimentales que han demostrado que la dosis utilizada es el principal determinante de la curación ${ }^{3}$. Si bien nuestro caso clínico corresponde a una "cepa de área templada", es muy difícil a partir de un único caso sugerir recomendaciones sobre la utilización de dosis más altas de PQ con las cuales el paciente no alcanzó la curación radical. Creemos necesario profundizar los estudios sobre factores dependientes del hospedero y del parásito que pueden asociarse con el fracaso del tratamiento radical y las recaídas. La falta de evidencia clínica concluyente en relación al mejor esquema terapéutico hace indispensa- ble realizar un adecuado seguimiento de los pacientes a fin de lograr la identificación y el tratamiento precoz de las recaídas.

Agradecimientos. A la Sección de Parasitología del Hospital de Enfermedades Infecciosas "Dr. Francisco Javier Muñiz".

\section{Resumen}

La infección por Plasmodium vivax se caracteriza por la formación de hipnozoítos que permanecen quiescentes en los hepatocitos del hospedero y son responsables de las recaídas de la malaria. Primaquina es el único fármaco en uso para la erradicación de los hipnozoítos y asociado a un agente esquizonticida, constituye el tratamiento radical. Las fallas al tratamiento radical están relacionados con una dosificación subóptima, adherencia inadecuada y reinfección. Sin embargo, cuando estos factores están ausentes, se han postulado mecanismos propios del metabolismo del hospedero y de tolerancia del parásito. Se describe un caso de malaria por $P$. vivax adquirido en la región amazónica asistido fuera de la zona endémica, con múltiples recaídas a pesar del tratamiento radical adecuado.

\section{Referencias bibliográficas}

1.- Guerra C A, Howes R E, Patil A P, Gething P W, Van Boeckel T P, Temperley W H, et al. The international limits and population at risk of Plasmodium vivax transmission in 2009. PLoS Negl Trop Dis 2010; 4: e774.

2.- Cogswell F B. The hypnozoite and relapse in primate malaria. Clin Microbiol Rev 1992; 5: 26-35.

3.- Guidelines for the treatment of malaria. 3rd edition. World Health Organization; 2015. Disponible en http://www.who.int/malaria/ publications/atoz/9789241549127/en/ (Fecha acceso: 12 de abril de 2016).

4.- Vásquez-Vivar J, Augusto O. Hydroxylated metabolites of the antimalarial drug primaquine. J Biol Chem 1992; 267: 6848-54.

5.- Baird J K. Resistance to therapies for infection by Plasmodium vivax. Clin Microbiol Rev 2009; 22: 508-34.

6.- $\quad$ Chiang T Y, Lin W C, Kuo M C, Ji D D, Fang C T. Relapse of imported vivax malaria despite standard-dose primaquine therapy: an investigation with molecular genotyping analyses. Clin Microbiol Infect 2012; 18: E232-4.

7.- Smoak B L, DeFraites R F, Magill A J, Kain $\mathrm{K}$ C, Wellde B T. Plasmodium vivax infections in US Army troops: failure of primaquine to prevent relapse in studies from Somalia. Am J
Trop Med Hyg 1997; 56: 231-4.

8.- Townell N, Looke D, McDougall D, McCarthy J S. Relapse of imported Plasmodium vivax malaria is related to primaquine dose: a retrospective study. Malar J 2012; 11: 214.

9.- Kristensen K, Dragsted U B. Recurrent Plasmodium vivax malaria due to dosedependent primaquine resistance: a case report. Scand J Infect Dis 2014; 46 (1): 63-5.

10.- Goller J L, Jolley D, Ringwald P, Biggs B A. Regional differences in the response of Plasmodium vivax malaria to primaquine as anti-relapse therapy. Am J Trop Med Hyg 2007; 76: 203-7.

11.- Arias A E, Corredor A. Low response of Colombian strains of Plasmodium vivax to classical antimalarial therapy. Trop Med Parasitol 1989; 40: 21-3.

12.- Duarte E C, Pang L W, Ribeiro L C, Fontes C J. Association of subtherapeutic dosages of a standard drug regimen with failures in preventing relapses of vivax malaria. Am J Trop Med Hyg 2001; 65: 471-6.

13.- da Silva A R, Carneiro E W, Dos Santos H J. Resposta dos plasmódios humanos aos antimaláricos na ilha de São Luís, estado do Maranhão, Brasil. Rev Inst Med Trop Sao Paulo 1984; 26: 139-46.

14.- Queensland Notifiable Disease Report 20022006: Queensland Health Communicable Disease Unit. 2007: 184-187. Disponible en https://www.health.qld.gov.au/ph/documents/ cdb/notif_dis_report0206.pdf (Fecha acceso: 12 de abril de 2016).

15.- Krudsood S, Tangpukdee N, Wilairatana $P$, Phophak N, Baird J K, Brittenham G M, et al. High-dose primaquine regimens against relapse of Plasmodium vivax malaria. Am J Trop Med Hyg 2008; 78: 736-40.

16.- Galappaththy G N, Omari A A, Tharyan P. Primaquine for preventing relapses in people with Plasmodium vivax malaria. Cochrane Database Syst Rev 2007; 24 (1): CD004389.

17.- Baird J K, Hoffman S L. Primaquine therapy for malaria. Clin Infect Dis 2004; 39: 1336-45.

18.- Pybus B S, Sousa J C, Jin X, Ferguson J A, Christian R E, Barnhart R, et al. CYP450 phenotyping and accurate mass identification of metabolites of the 8-aminoquinoline, antimalarial drug primaquine. Malar J 2012; 11 : 259.

19.- Ganesan S, Tekwani B L, Sahu R, Tripathi L M, Walker L A. Cytochrome P (450)-dependent toxic effects of primaquine on human erythrocytes. Toxicol Appl Pharmacol 2009; 241: 14-22.

20.- Ganesan S, Chaurasiya N D, Sahu R, Walker L A, Tekwani B L. Understanding the mechanisms for metabolism-linked hemolytic toxicity of primaquine against glucose 6-phosphate dehydrogenase deficient human erythrocytes: evaluation of eryptotic 
pathway. Toxicology 2012; 294: 54-60.

21.- Bennett J W, Pybus B S, Yadava A, Tosh D, Sousa J C, Mc Carthy W F, et al. Primaquine failure and cytochrome P-450 2D6 in Plasmodium vivax malaria. New Engl J Med 2013; 369: 1381-2.

22.- Centers for Disease Control and Prevention. Guidelines for treatment of malaria in the United States. 2013. Disponible en http://www. cdc.gov/malaria/resources/pdf/treatmenttable. pdf (Fecha de acceso 12 de abril de 2016)

23.- Lalloo D G, Shingadia D, Bell D J, Beeching N J, Whitty C J, Chiodini P L. UK malaria treatment guidelines 2016. J Infect 2016; 72 : 635-49.

24.- Rajgor D D, Gogtay N J, Kadam V S, Kocharekar M M, Parulekar M S, Kshirsagar $\mathrm{N}$ A, et al. Antirelapse efficacy of various primaquine regimens for Plasmodium vivax. Malar Res Treat 2014; 2014: 347018.
25.- Bright A T, Alenazi T, Shokoples S, Tarning J, Paganotti G M, White N J, et al. Genetic analysis of primaquine tolerance in a patient with relapsing vivax malaria. Emerg Infect Dis 2013; 19: 802-5.

26.- Kublin J G, Dzinjalamala F K, Kamwendo D D, Malkin E M, Cortese J F, Martino L $\mathrm{M}$, et al. Molecular markers for failure of sulfadoxinepyrimethamine and chlorproguanildapsone treatment of Plasmodium falciparum malaria. J Infect Dis 2002; 185: 380-8.

27.- Mu J, Ferdig M T, Feng X, Joy D A, Duan J, Furuya T, et al. Multiple transporters associated with malaria parasite responses to chloroquine and quinine. Mol Microbiol 2003; 49: 977-89.

28.- Reed M B, Saliba K J, Caruana S R, Kirk K, Cowman A F. Pgh1 modulates sensitivity and resistance to multiple antimalarials in Plasmodium falciparum. Nature 2000; 403: 906-9.
29.- Hill D R, Baird J K, Parise M E, Lewis L S, Ryan E T, Magill A J. Primaquine: report from $\mathrm{CDC}$ expert meeting on malaria chemoprophylaxis I. Am J Trop Med Hyg 2006; 75: 402-15.

30.- Raj D K, Mu J, Jiang H, Kabat J, Singh S, Sullivan M, et al. Disruption of a Plasmodium falciparum multidrug resistance-associated protein (PfMRP) alters its fitness and transport of antimalarial drugs and glutathione. J Biol Chem 2009; 284: 7687-96.

31.- Neafsey D E, Galinsky K, Jiang R H, Young L, Sykes S M, Saif S, et al. The malaria parasite Plasmodium vivax exhibits greater genetic diversity than Plasmodium falciparum. Nat Genet 2012; 44: 1046-50.

32.- Collins W E, Jeffery G M. Primaquine resistance in Plasmodium vivax. Am J Trop Med Hyg 1996; 55: 243-9. 